\title{
Real-time observation of pathophysiological processes during murine experimental Schistosoma japonicum infection using high-resolution ultrasound imaging
}

Katsumi Maezawa ${ }^{1 *}$ (D), Rieko Furushima-Shimogawara ${ }^{1}$, Akio Yasukawa ${ }^{2}$, Nobuo Ohta ${ }^{1,3}$ and Shiro Iwanaga ${ }^{1}$

\begin{abstract}
Background: Hepatosplenic lesion formation is one of the typical clinical symptoms of schistosomiasis japonica. Although it is established that circum-oval granuloma formation mediated by T lymphocytes is the key event triggering the formation of hepatic lesions, the time-course kinetics of disease progression remains to be fully elucidated.

Methods: The real-time process of the pathophysiology of schistosomiasis japonica from the early to late clinical phase was non-invasively observed in a murine experimental infection model using high-resolution ultrasonography. Together with clinical parameters, including body weight and the levels of serum markers of hepatic damage or fibrosis, ultrasonography was used to assess changes in the liver parenchyma and diameter of the portal vein and portal blood flow velocity. In parallel, parasitological parameters were observed, including egg number in the feces and maturation of parasites.

Results: Abnormal high-echo spot patterns in the liver parenchyma, reflecting hepatic fibrosis in ultrasonography, appeared in the liver at 4 weeks post-infection and the pattern became more enlarged and severe over time. This finding was concordant with parasite maturation and initial egg excretion. The serum M2BPGi level markedly increased from 8 weeks post-infection, suggesting sharp deterioration of hepatic fibrosis. At the same time, the diameter of the portal vein, reflecting portal hypertension, became enlarged and reached the peak level at 8 weeks post-infection. Ascites were apparent around the spleen at 9 weeks post-infection, and dilatation of the splenic vein was noted at 10 weeks postinfection. Live adult worms seemed to be detected in the portal vein at 4 weeks post-infection by ultrasonography.

Conclusions: We obtained real-time imaging of the development of hepatosplenic lesions of schistosomiasis japonica in mice. The time-course kinetics of the onset, development, and modulation of each symptom was uncovered. These results are expected to provide new clues for understanding the pathophysiology of human schistosomiasis japonica.
\end{abstract}

Keywords: Schistosoma japonicum, Schistosomiasis, Ultrasonography, Non-invasive observation, Liver fibrosis, Portal hypertension, Real-time imaging

\footnotetext{
* Correspondence: yrl00232katsumi@gmail.com

${ }^{1}$ Department of Environmental Parasitology, Tokyo Medical and Dental

University Graduate School of Medical and Dental Sciences, 1-5-45 Yushima,

Bunkyo-ku, Tokyo 113-8519, Japan

Full list of author information is available at the end of the article
} 


\section{Background}

Schistosomiasis is caused by the intravenous trematode Schistosoma sp., and is endemic to more than 70 countries, putting 218 million people at risk of infection. Schistosoma japonicum infection is endemic in Asian countries, including China, the Philippines, and Indonesia; as a zoonotic pathogen, control of S. japonicum infection is more complicated and difficult compared with that of other schistosome species [1, 2]. Schistosomiasis is divided into two clinical types, intestinal and urinary, and S. japonicum causes the former type. Schistosomiasis japonica is characterized by severe hepatosplenic lesions with circum-oval granuloma formation, hepatic fibrosis, portal hypertension, and so forth [3-5].

The immunopathology of schistosomiasis has been well characterized; several studies have shown that the $\mathrm{T}$ cell response to egg antigens triggers the symptoms. Parasite eggs discharged inside blood vessels are trapped in the capillary vessels, which induces granuloma formation around the vessels under the control of egg antigen-specific $\mathrm{T}$ cell responses [6-8]. Cercariae, the infective larvae of schistosome parasites, penetrate the host skin and mature in the mesenteric/portal vein. In a murine experimental infection model, adult S. japonicum start egg production around 4 weeks post-infection (PI), and thus, the hepatosplenic lesion develops as of 4 weeks PI [9]. The peak response is observed around 8 weeks PI, and then, the granulomatous response is subsequently downregulated due to initiation of an immunomodulation mechanism [10]. Discussion on the time-course development of pathology has mainly been based on cross-sectional observations because the samples were obtained from sacrificed animals at the time of observation. However, continuous changes during the infection process have not yet been studied because of the lack of available non-invasive equipment for small experimental animals. High-resolution ultrasonography is a recently available technology, which has now become applicable for studies with mice [11, 12]. As a non-invasive approach, it is possible to conduct continuous observations of disease progress in the same mouse using high-resolution ultrasonography $[13,14]$. There is no direct evidence that the clinicopathological changes observed in a mouse model are comparable to those in human infection; however, the pathophysiology of schistosomiasis in humans seems to be similar to findings in the murine model. Thus, research on the pathophysiology of human schistosomiasis would be greatly promoted with the possibility to conduct longitudinal, but not cross-sectional, observations in mice.

Accordingly, the aim of the present study was to conduct real-time observations of the progress of schistosomiasis japonica using high-resolution ultrasonography in a murine model of infection. The main goal of our study was to continuously observe the time-course development of hepatosplenic lesions in a non-invasive manner in the same group of mice to obtain more detailed and dynamic information on the pathophysiology of schistosomiasis japonica. The results were compared with information obtained by conventional parasitological and/or pathological tools. Based on this new source of information, we discuss the process of disease development and modulation and the future applicability of this approach.

\section{Methods \\ Animals}

Female BALB/c mice (5 weeks old) were used in this study (Crea Japan, Tokyo). The mice were maintained at $23{ }^{\circ} \mathrm{C}$ with a 12 -h light/12-h dark cycle with free access to food and water.

\section{Parasite infection}

For short to mid-term observations up to 13 weeks PI (group 1, $n=12$ ), 5-week-old mice (body weight 18$21 \mathrm{~g})$ were infected with 25 cercariae of the Yamanashi strain of S. japonicum under intraperitoneal anesthesia with $50 \mathrm{mg} / \mathrm{kg}$ of pentobarbital as described elsewhere [15]. In parallel, another group of mice (group 2, $n=10$ ) was infected with 10 cercariae for longer term observation for up to 1 year. Mice that were not infected were used as negative controls.

\section{Body weight and egg number per gram (EPG) counts}

Body weight of the infected mice of group 1 was measured up to 13 weeks PI except for 3 weeks PI and 6 weeks PI. Worm eggs were gathered from the mice feces of each week in order, fixed the eggs with formalin, melted the residue with ethyl acetate, and then collected by MDL modification method in which the eggs were precipitated by centrifugation. Thereafter, the number of the eggs per $1 \mathrm{~g}$ in feces was calculated by microscopic examination (EPG). EPG was determined at 7, 8, 9, and 11 weeks PI.

\section{Ultrasonographic observation}

Ultrasonic examination was continuously performed weekly for weeks from 3 to 13 weeks, randomly selected from group 1 . For the mice randomly selected, the same individuals were used until the end of the experiment to see changes in intraperitoneal cavity and EPG over time. The mice were fixed on a platform after being anesthetized with Somnopentyl ${ }^{\circledR}$ or Isoflurane ${ }^{\ominus}$, and ultrasonographic observation was performed while keeping the mouse in a fixed position with a conditioned temperature and stable circulation. The echo jelly warmed with hot water was applied to the same site, and 
the optimal position for intraperitoneal retrieval was used. For the mice in group 2, the observation was performed at 1 year PI.

We used an ALOKA Noblus (linear probe for humans, 18-4 MHz; Hitachi Corporation, Tokyo, Japan) or Prospect 3.0 ultrasound imaging apparatus for small animals (50 $\mathrm{MHz}$, resolution $30 \mu \mathrm{m}$; Nepagene, Chiba). The ALOKA machine was used for observations over a wide range of tissues, including the liver, spleen, portal vein, intestine, and ascites. The spleen depth was measured in the middle portion of the organ. The diameter of the portal vein trunk was measured at the porta hepatis, and the diameter of the splenic vein was measured near the branch from the portal vein. The Prospect 3.0 machine was used for measuring the angle of the liver edge and portal blood flow.

\section{Blood markers of liver function and fibrosis}

A mouse different from the mouse used in the ultrasonic examination was sacrificed by injection of a lethal dose of pentobarbital. Blood samples were obtained at $0,6,7$, $8,10,13$, and 23 weeks PI.

After macroscopic aspects of the livers and other abdominal organs were recorded, blood was collected from the posterior vena cava and the alanine amino transferase (ALT), albumin/globulin ratio (A/G), and Mac-2 binding protein glycosylation isomer (M2BPGi) [16-18] values were measured as indicators of liver function and hepatic fibrosis. ALT and A/G were inspected by Oriental Yeast Co., Tokyo and M2BPGi was inspected with Possible Co., Gifu.

\section{Statistical analysis}

Statistical analysis was performed with Student's $t$ test. $P$ values $<0.05$ were regarded as statistically significant.

\section{Results}

\section{Body weight and EPG}

There was no difference in body weight up to 6 weeks PI, but differences in body weight became apparent between infected mice and control mice as of 7 weeks PI (Fig. 1). Although the difference was only statistically marginal, this trend suggests the progression of general symptoms as of 6 weeks PI. An increase of EPG was also observed on the infected mice at 7 weeks PI, and EPG continuously increased over time (Fig. 1).

\section{Blood parameters}

The ALT values increased as of 6 weeks PI, and the maximum values were noted around 7 weeks PI. The M2BPGi level, as a marker of hepatic fibrosis, was increased from 8 weeks PI. After that, it decreased markedly at 13 weeks, again high at 23 weeks. The A/G ratio decreased gradually up to 6 weeks PI and was stable thereafter. Together, these results suggested that failure of liver function was most apparent around 68 weeks PI (Fig. 2).

\section{Ultrasonography \\ Liver}

In the control mice, there was no particular signal in the liver, and the strength of the signal was almost at the same level between the liver and kidney through the study period. In group 1 mice, no particular change was detected before 3 weeks PI, but diffuse spots of a highecho signal were detected at 4 weeks PI and after. These spots then increased in number, a thin linear shadow appeared at 8 weeks PI, and the spots were fused to each other and expanded in size at 10 weeks PI. These linear high-echo signal areas became more intensified and spread over the whole liver. A similar tendency was

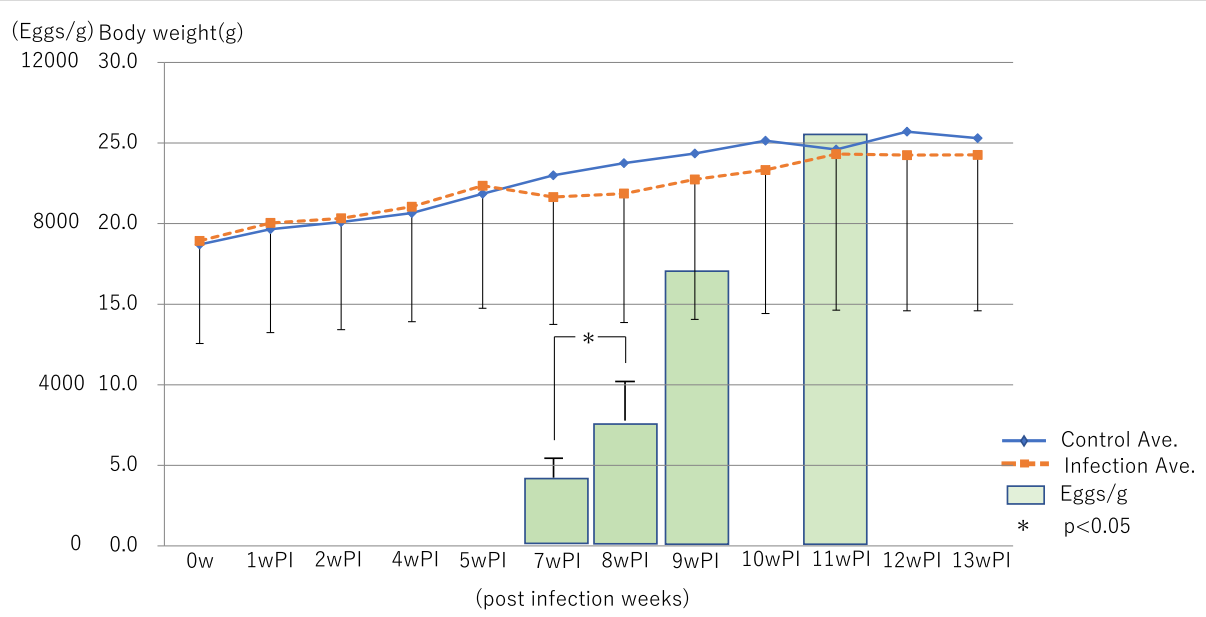

Fig. 1 Observation of body weight and EPG. Dotted line lines indicate changes in body weight of infected mice and solid line lines indicate control mice. The bars showed EPG. Differences in body weight seemed to be apparent between infected mice and control mice after 7 weeks PI. However, there was no significant difference between control and infected mice 


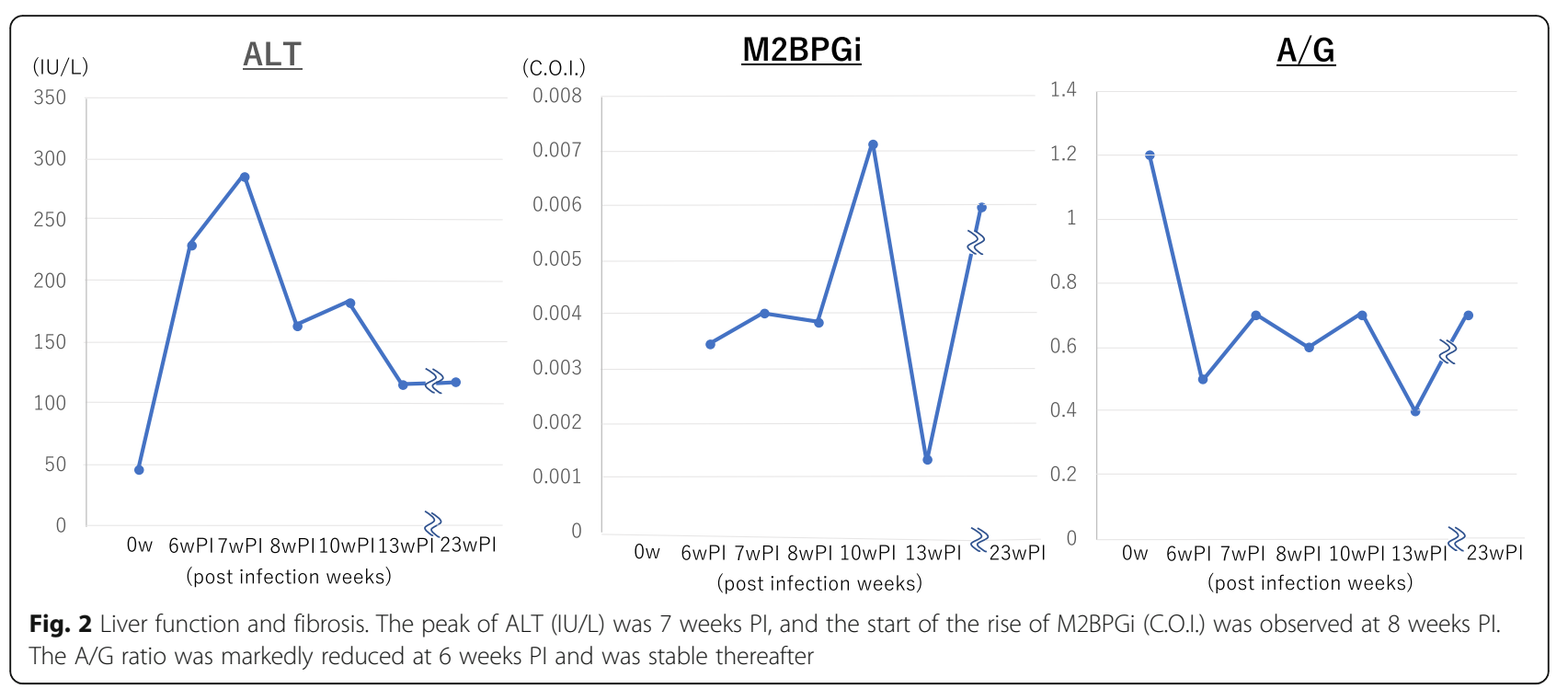

detected in the livers of group 2 mice, and the linear high-echo spots were even further intensified (Fig. 3).

Along with the progression of infection, the angle of the periphery of the liver became gradually obtuse, indicating the appearance of hepatic hypertrophy and/or atrophy (Fig. 4).

\section{Portal vein and splenic vein}

The diameter of the portal vein of the control mice was $0.7-0.8 \mathrm{~mm}$, which was enlarged in group 1 mice. Such enlargement continued until 8 weeks PI, but then gradually recovered to the normal range. The mice in group 2 (at 1 year PI) showed a portal vein diameter that was similar to that of the control mice. The blood flow of the portal vein temporally slowed down at 6 weeks PI, but returned back to the control level thereafter (Fig. 5). Of note, an unidentified image was observed inside the portal vein at 4 weeks PI and thereafter. The image was independent of the heart beat and moved slowly, suggesting that it was a live worm body.

The diameter of the splenic vein was approximately $0.3 \mathrm{~mm}$ in control mice at the site of divergence from the portal vein and then doubled by 10 weeks PI (Fig. 6). A similar degree of dilatation was observed at 12 weeks PI, but it was difficult to clearly detect in the mice at 1 year PI due to the influence of intestinal flatulence.

\section{Other findings of the peritoneal cavity}

The depth of the spleen became gradually enlarged from 4 weeks PI (Fig. 7). A low level of ascites was noted at

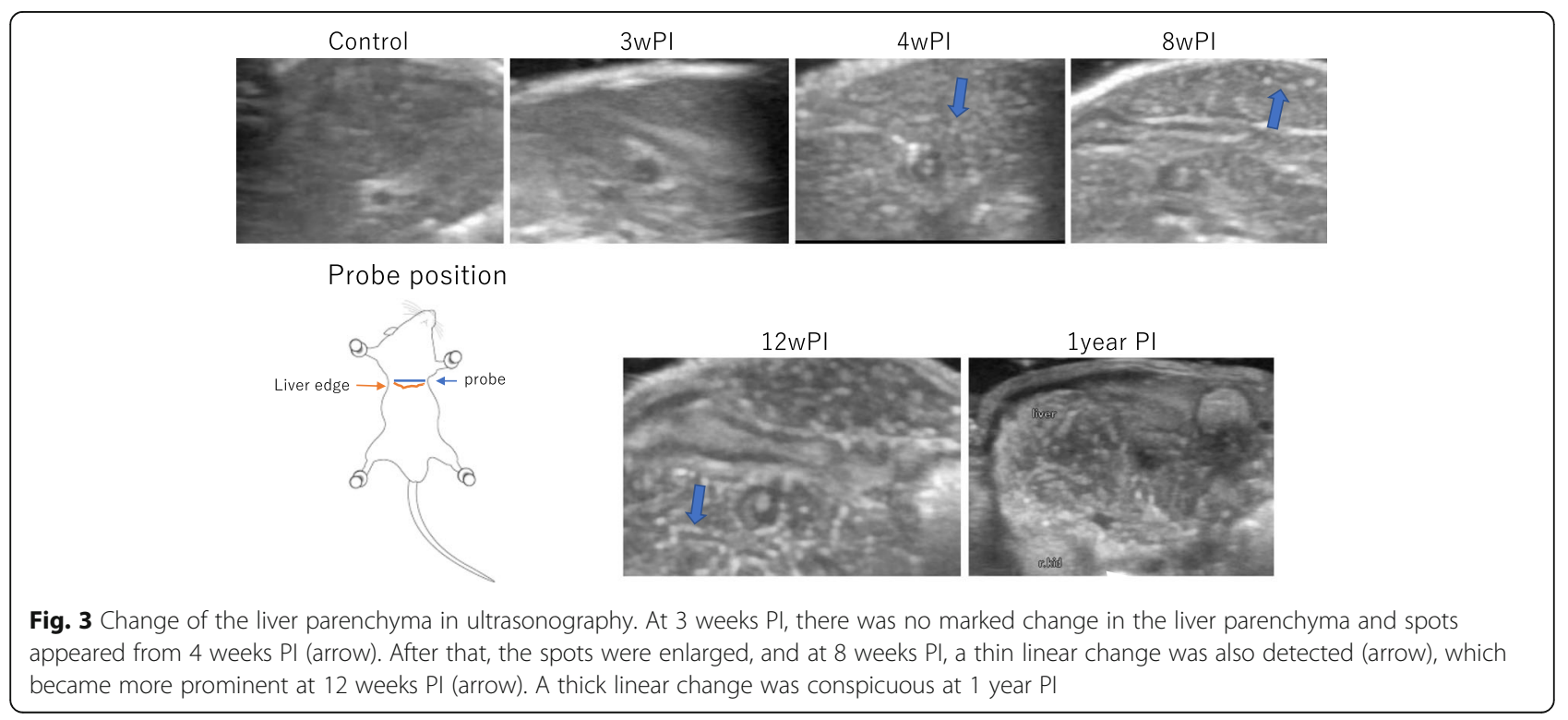




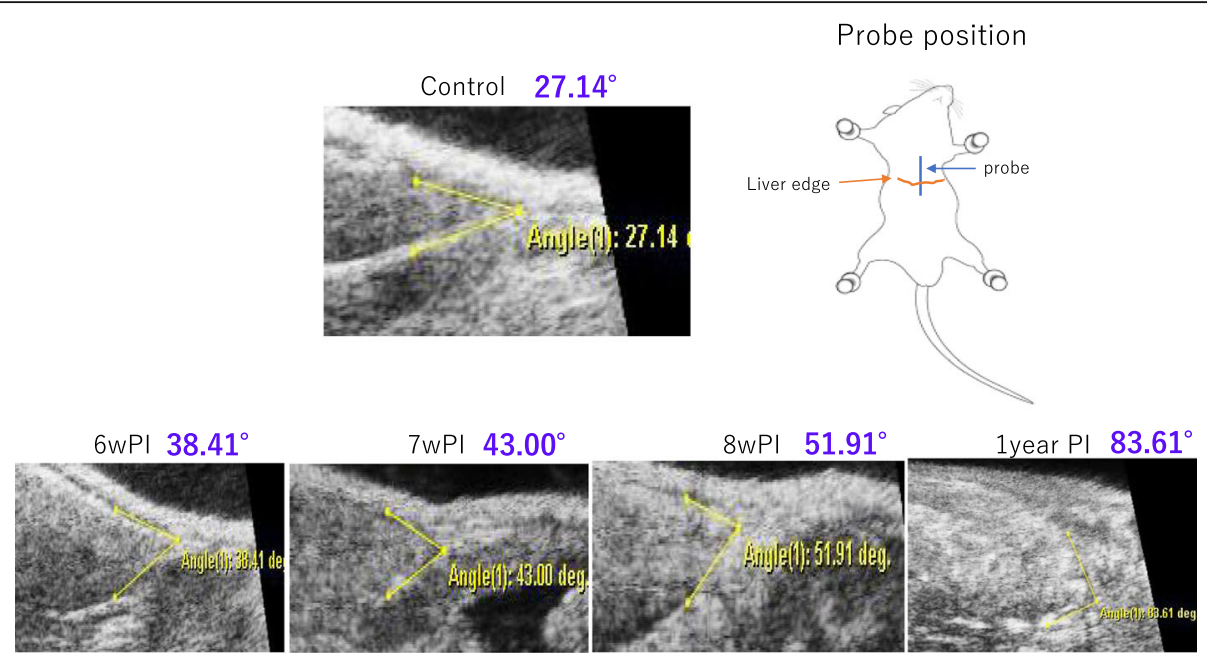

Fig. 4 Angle of the liver edge. The angle of the periphery of the liver became gradually obtuse

9 weeks PI, which increased gradually at 10 weeks PI and thereafter. The mice at 1 year PI showed more intense ascites (Fig. 8). Observation of the intestinal wall suggested loss of flexibility as of 6 weeks PI because a high-echo signal was noted in the intestinal wall thickening, which increased in intensity over time (Fig. 9).

\section{Discussion}

Recent developments of tools for bioimaging have provided the opportunity to conduct real-time observations of various biological phenomena and track the responses occurring inside living bodies [19, 20]. High-resolution ultrasonography is one such tool for real-time bioimaging and has been applied in various fields of clinical medicine for diagnosis and/or clinical evaluation. Highresolution ultrasonography is already widely applied in the field of veterinary medicine, and improvement of the tools has facilitated their application in experimental mice for research.

The profiles of parasitic diseases are outcomes of complicated host-parasite interactions and require continuous/longitudinal observation rather than conventional cross-sectional observations, to provide information for gaining a more profound understanding of the pathophysiology. Although it has been elucidated that hepatic fibrosis, portal hypertension, splenomegaly, ascites, and other characteristics are typical findings of schistosomiasis japonica [21-23], the underlying pathophysiology remains somewhat of a jigsaw puzzle because each piece appears to be somewhat independent and this information has been obtained using different sample sources and approaches.
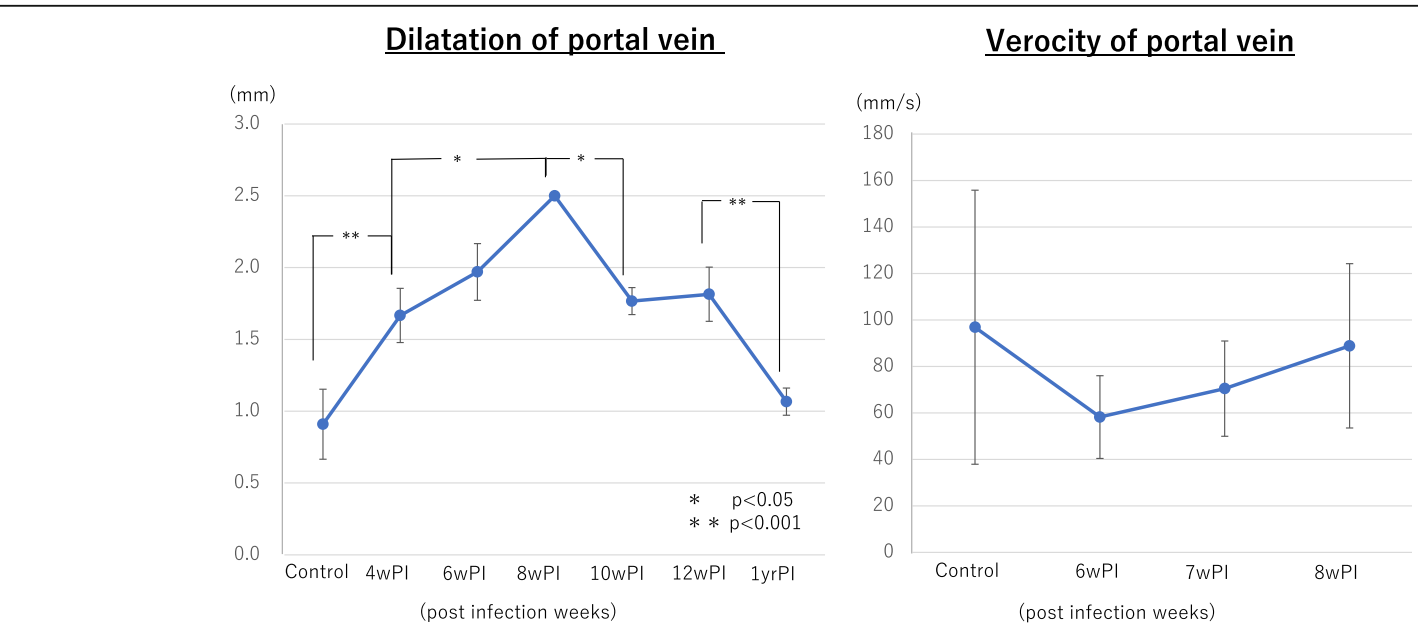

Fig. 5 Dilatation of the portal vein and velocity. The portal vein became enlarged, which continued until 8 weeks PI and then gradually recovered to the normal range. Blood flow of the portal vein temporally slowed down at 6 weeks PI but returned back to the control level thereafter 


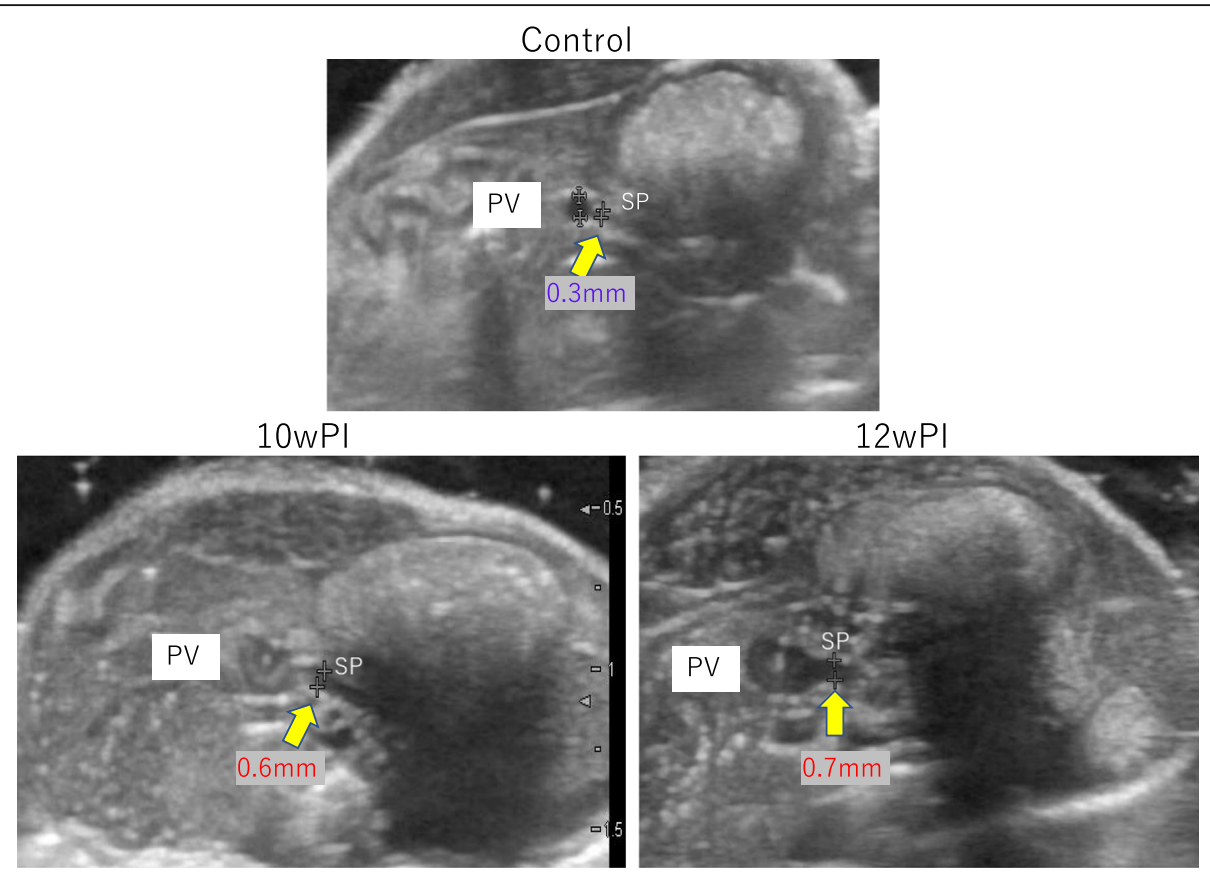

Fig. 6 Dilatation of the splenic vein. The diameter of the splenic vein (SP) was around $0.3 \mathrm{~mm}$ in control mice at the site of divergence from the portal vein (PV) but doubled in size at 10 and 12 weeks PI

In the present study, we adopted high-resolution ultrasonography to determine the time-course changes of hepatosplenic lesions formed during S. japonicum infection in mice, and the findings and their detailed order of each clinical sign are summarized in Fig. 10. In brief, liver dysfunction due to embolized eggs was the first response, followed by hepatic fibrosis. Hepatic fibrosis was enhanced without modulation, and thickening of the intestinal wall appeared at around the same time or slightly earlier. The thickening of the intestinal wall is more likely to be due to embolism by worm eggs [24, 25]. Portal hypertension was observed later, and the peak level was detected at around 8 weeks PI. Ascites and dilatation of the splenic vein were the final events detected; after that time point, the portal hypertension and liver function impairment returned to the control levels.

Hepatic fibrosis is one of the typical symptoms of schistosomiasis japonica. Spotty echo was detected in the liver parenchyma of infected mice at 4 weeks PI and after, which was similar to the "starry-sky"-like pattern of changes in the human liver described by the World Health Organization (WHO) [26-28]. In the WHO guidelines, the extent of liver parenchyma in

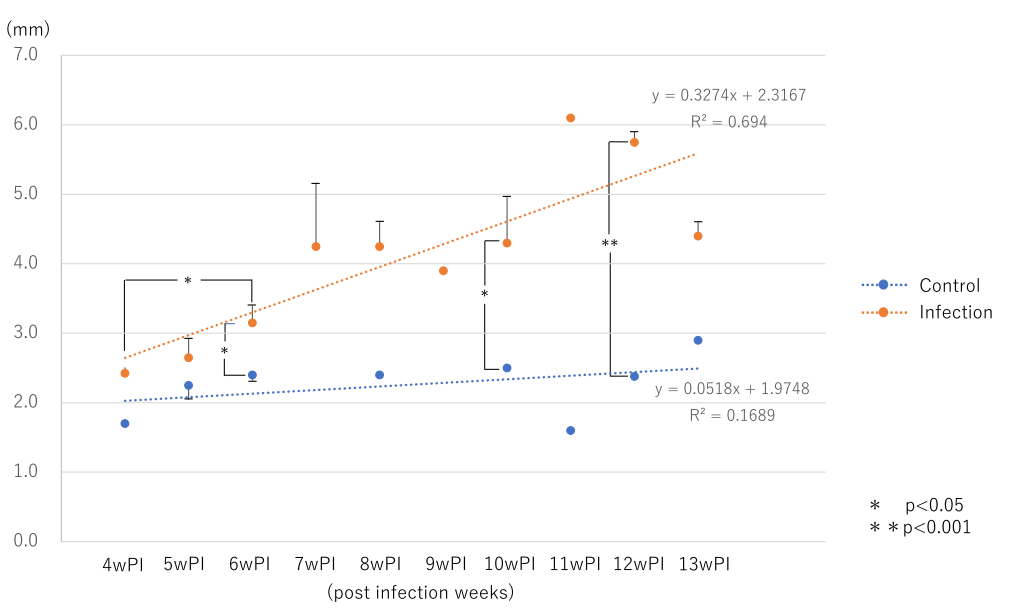

Fig. 7 Depth of the spleen. The depth of the spleen was gradually enlarged from 4 weeks PI and then continued to increase 


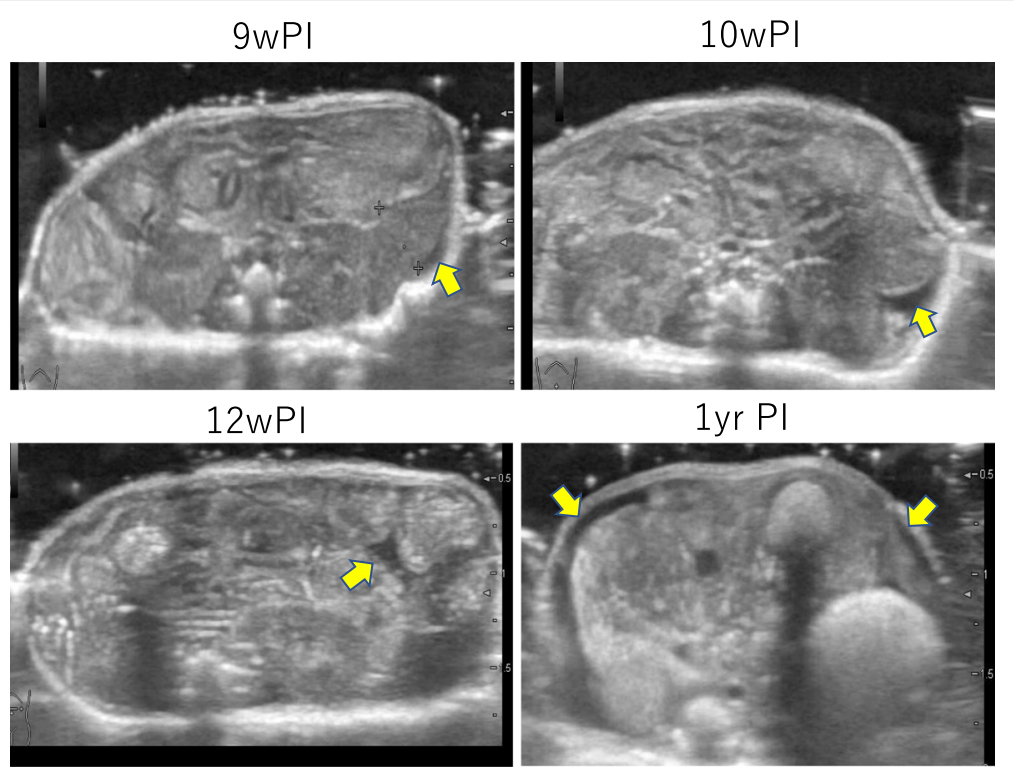

Fig. 8 Ascites. Low-level ascites were noted at 9 weeks PI and increased gradually at 10 weeks PI and thereafter. Mice at 1 year PI showed more intense ascites. (arrow; ascites)

ultrasonography is scored and used for evaluating the severity of schistosomiasis japonica, in the order of a "starry-sky," "rings and pipe-stems," "ruff" around portal bifurcation, "patches," and "bird's claw" pattern. In the present study, starry-sky-like lesions were observed as of 4 weeks PI, which might indicate egg granuloma formation and fibrosis of Glisson's sheath [9]. This finding was concordant with the timing of oviproduction and egg embolism in the microcapillary vessels in Glisson's sheath. The fibrotic pattern observed in the late phase in mice seemed to be equivalent to the "network pattern" of fibrosis observed in cases of human schistosomiasis [29-31]. Our observation of mice at 1 year PI revealed more severe fibrotic patterns, although the other parameters returned to almost normal levels.
Along with hepatic fibrosis, hypertrophy or atrophy of the liver was promoted, suggesting that the liver damage had already been promoted in the earlier phase of infection. The liver dysfunction was caused by microembolism of the eggs, resulting in a circulatory disturbance, and hepatic function was impaired from the beginning of infection. Although liver function was impaired during the phase of hepatic fibrosis, it recovered in spite of the continuous progression of hepatic fibrosis. Progress of hepatic fibrosis on ultrasound images was not a parallel trend to the transition of M2BPGi level. M2BPGI level may be similar to markers such as IL-17 and TGF$\beta[9]$.

With regard to portal hypertension, the hepatic arterial blood flow rate has been reported to increase to

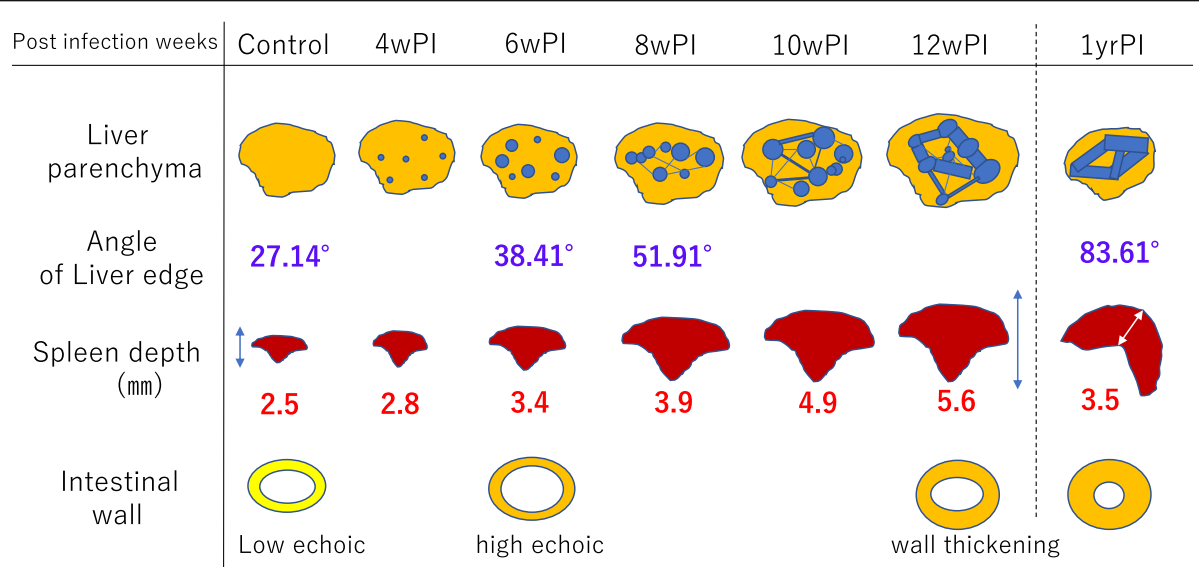

Fig. 9 Schema of hepatosplenomegaly and intestinal lesions. Along with changes in the liver parenchyma and changes in the spleen crosssection, a high-echo signal was noted indicating intestinal wall thickening, which increased in intensity over time 


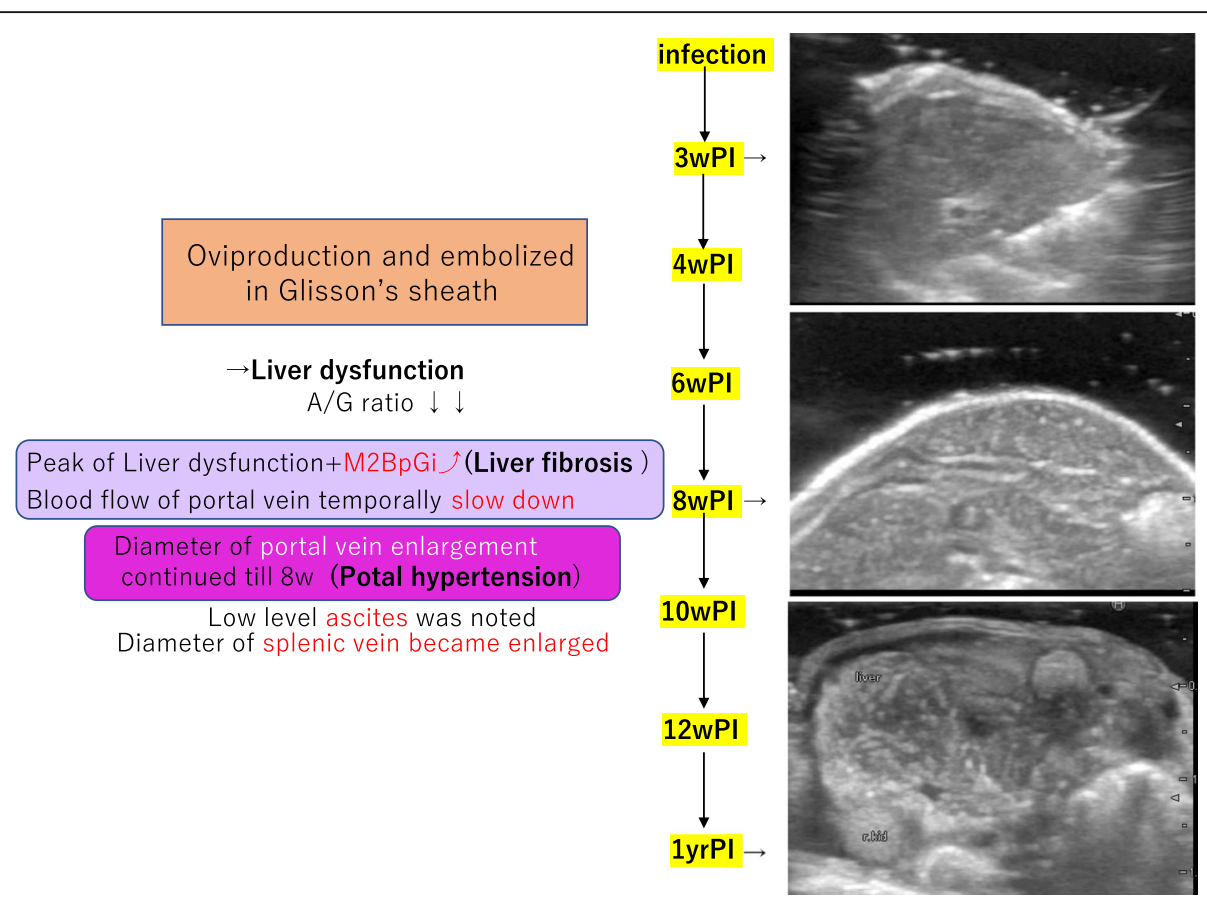

Fig. 10 Time course of an experimental mouse model of S. japonicum infection. We were able to capture the progression of hepatic parenchyma abnormalities, secondary liver fibrosis, and portal hypertension over time with the support of blood data

compensate for the decreased portal blood flow [32-34]; however, this was not apparent in our present observations. The diameter and blood flow of the portal vein suggested that the diameter and portal pressure were associated in the early phase of infection. However, hepatic fibrosis was at the maximum level around 8 weeks PI and then gradually decreased, reaching almost normal levels (despite the presence of severe hepatic fibrosis) at 1 year PI. There are various explanations for this finding. For example, portal hypertension reached the maximum level at the time point of maximum egg granuloma formation, or blood flow continued through bypass routes to eliminate the portal hypertension. As the dilatation of the splenic vein was clearly detected at 10 weeks PI, a change in hemodynamics similar to that of human portal hypertension could have occurred, which was not captured on the ultrasound during our observations. It is thus possible to speculate that portal hypertension and the degree of hepatic fibrosis are not necessarily parallel responses to infection.

It is interesting to note that our high-resolution ultrasonographic observation seemed to detect the presence of a live parasite in the portal vein. The image moved spontaneously and slowly, and the length was comparable to the size of adult $S$. japonicum. If this image was indeed an adult worm, and if it would be possible to obtain more clear images, high-resolution ultrasonography can emerge as a new diagnostic approach. From the perspective of parasitology, it is still not clear how the schistosomulae move from the host lung to the mesenteric/portal vein. Thus, real-time bioimaging might be a useful tool for resolving this long-standing question.

The real-time bioimaging of the pathophysiology of schistosomiasis japonica can also provide a new tool for research on vaccine/new drug development. Currently, the efficacies of a vaccine and/or new drug with respect to reduction in the worm burden or alleviation of the pathological damage are analyzed with conventional approaches. It is expected that new information would become available by conducting imaging analysis with ultrasonography, especially with respect to how and when the worms are killed or the pathology becomes modified in experimental murine schistosomiasis. This would further help to expand strategies of vaccine/drug development and make such control tools more feasible in the near future [35].

\section{Conclusions}

We successfully conducted the real-time imaging of the development of hepatosplenic lesions of schistosomiasis japonica in mice. It is expected that new information will become available using imaging analysis with ultrasonography as to how and when the worms are killed or the pathology is modified in experimental murine schistosomiasis. 


\section{Abbreviations}

A/G: Albumin/globulin ratio; ALT: Alanine amino transferase; EPG: Egg number per gram of feces; M2BPGi: Mac-2 binding protein glycosylation isomer; PI: Post-infection

\section{Acknowledgements}

The authors give thanks to Dr. A. Hino for her suggestions and technical advice. Also, I would like to express my gratitude to Dr. K. Uemura for providing us with equipment.

\section{Funding}

Not applicable

\section{Availability of data and materials}

The datasets used and/or analyzed during the current study are available from the corresponding author on reasonable request.

\section{Authors' contributions}

KM and RFS conceived and designed the study. AY contributed to the ultrasonographic analysis. NO and SI were involved in planning the study and data analysis. All authors read and approved the final manuscript.

\section{Ethics approval and consent to participate}

The animal experiment in this study was approved by the Committee of Tokyo Medical and Dental University (\#81262).

\section{Consent for publication}

Not applicable

\section{Competing interests}

The authors declare that they have no competing interests.

\section{Publisher's Note}

Springer Nature remains neutral with regard to jurisdictional claims in published maps and institutional affiliations.

\section{Author details}

'Department of Environmental Parasitology, Tokyo Medical and Dental University Graduate School of Medical and Dental Sciences, 1-5-45 Yushima, Bunkyo-ku, Tokyo 113-8519, Japan. ${ }^{2}$ Nishiogi Veterinary Medical Hospital, 4-9-2 Nishiogikita, Suginami-ku, Tokyo 167-0042, Japan. ${ }^{3}$ Depertment of Clinical Nutrition, Faculty of Health Science, Suzuka University of Medical Science, 1001-1, Kishioka-cyo, Suzuka-shi, Mie 510-0293, Japan.

\section{Received: 8 November 2017 Accepted: 19 December 2017}

Published online: 05 January 2018

\section{References}

1. Chitsulo L, Engels D, Montresor A, Savioli L. The global status of schistosomiasis and its control. Acta Trop. 2000:77:41-51.

2. Fernandez TJ Jr, Pettila T, Banez B. An epidemiological study on Schistosoma japonicum in domestic animals in Leyte, Philippines. Southeast Asian J Trop Med Public Health. 1982:13:575-9.

3. Barsoum RS, Esmat G, El-Baz T. Human schistosomiasis: clinical perspective: review. J Adv Res. 2013:4:433-44.

4. Weerakoon KGAD, Gobert GN, Cai P, McManus DP. Advances in the diagnosis of human schistosomiasis. Clin Microbiol Rev. 2015;28:939-67.

5. Zhang BB, Cai WM, Tao J, Zheng M, Liu RH. Expression of Smad proteins in the process of liver fibrosis in mice infected with Schistosoma japonicum. Chinese J Parasitol Parasit Dis. 2013;31:89-94. [Article in Chinese]

6. Bartley PB, Ramm GA, Jones MK, Ruddell RG, Li Y, McManus DP. A contributory role for activated hepatic stellate cells in the dynamics of Schistosoma japonicum egg-induced fibrosis. Int J Parasitol. 2006;36: 993-1001.

7. Wynn TA, Cheever AW. Cytokine regulation of granuloma formation in schistosomiasis. Curr Opin Immunol. 1995:7:505-11.

8. Wilson MS, Mentink-Kane MM, Pesce JT, Ramalingam TR, Thompson R, Wynn TA. Immunopathology of schistosomiasis. Immunol Cell Biol. 2007;85: 148-54.

9. Zhang Y, Chen L, Gao W, Hou X, Gu Y, Huang D, et al. IL-17 neutralization significantly ameliorates hepatic granulomatous inflammation and liver damage in Schistosoma japonicum infected mice. Eur J Immunol. 2012;42: 1523-35.

10. Jenkins SJ, Hewitson JP, Jenkins GR, Mountford AP. Modulation of the host's immune response by schistosome larvae. Parasite Immunol. 2005;27:385-93.

11. Foster FS, Zhang MY, Zhou YQ, Liu G, Mehi J, Cherin E, et al. A new ultrasound instrument for in vivo microimaging of mice. Ultrasound Med Biol. 2002;28:1165-72

12. Han A, Erdman JW, Simpson DG. Early detection of fatty liver disease in mice via quantitative ultrasound. IEEE International Ultrasonics Symposium. 2014, 2014; https://doi.org/10.1109/ULTSYM.2014.0589.

13. Fernández-Domínguez I, Echevarria-Uraga JJ, Gómez N, Luka Z, Wagner C, Lu SC, et al. High-frequency ultrasound imaging for longitudinal evaluation of non-alcoholic fatty liver disease progression in mice. Ultrasound Med Biol. 2011;37:1161-9

14. Chen W, Chen JY, Tung YT, Chen HL, Kuo CW, Chuang CH, et al. Highfrequency ultrasound imaging to evaluate liver fibrosis progression in rats and Yi Guan Jian herbal therapeutic effects. Evid Based Complement Altern Med. 2013;2013:302325.

15. Tucker MS, Karunaratne LB, Lewis FA, Freitas TC, Liang YS. Schistosomiasis, 103. Curr Protoc Immunol, Unitas. 2013;19(1) https://doi.org/10.1002/ 0471142735.im1901s28.

16. Hisashi N. Development of M2BPGi: a novel fibrosis serum glyco-biomarker for bchronic hepatitis/cirrhosis diagnostics. Expert Rev Proteomics. 2015;12: 683-93.

17. Wei B, Feng S, Chen E, Li D, Wang T, Gou Y, et al. M2BPGi as a potential diagnostic tool of cirrhosis in Chinese patients with hepatitis $B$ virus infection. J Clin Lab Anal. 2017; https://doi.org/10.1002/jcla.22261.

18. Xu H, Kong W, Liu L, Chi X, Wang X, Wu R, et al. Accuracy of M2BPGi, compared with Fibro Scan ${ }^{\oplus}$, in analysis of liver fibrosis in patients with hepatitis C. BMC Gastroenterol. 2017:17:62.

19. Marcello M, Emilia V, Giuliana S, Adelaide G, Giancarlo T, Andrea A, et al Morphological ultrasound microimaging of thyroid in living mice. Endocrinology. 2009;150:4810-5.

20. Hartono $\mathrm{S}$, Thng $\mathrm{CH}$, Chuang $\mathrm{KH}$, Hung $\mathrm{H}$, Koh TS. Dynamic contrastenhanced MRI of mouse liver: a feasibility study using a dual-input twocompartment tracer kinetic model. JBiomedical Science and Engineering. 2015;8:90-7

21. Gryseels B, Polman K, Clerinx J, Kestens L. Human schistosomiasis. Lancet. 2006:368:1106-18.

22. Dessein A, Arnaud V, He H, Li J, Dessein H, Hou X, et al. Genetic analysis of human predisposition to hepatosplenic disease caused by schistosomes reveals the crucial role of connective tissue growth factor in rapid progression to severe hepatic fibrosis. Pathol Biol. 2013:61:3-10.

23. Dunn MA, Kamel R. Hepatic schistosomiasis. Hepatology. 1981;1:653-61.

24. Aki T. Examination of the findings with intestinal wall thickening in patients of liver cirrhosis on ultrasonography. Japanese J Med Ultrasound Technol. 2014:39:359-66.

25. Strickland GT. Gastrointestinal manifestations of schistosomiasis. Gut. 1994 35:1334-7.

26. Silva LCS, Andrade LM, Queiroz LC, Voieta I, Azeredo LM, Antunes CM, et al. Schistosoma mansoni magnetic resonance analysis of liver fibrosis according to WHO patterns for ultrasound assessment of schistosomiasis-related morbidity. Mem Inst Oswaldo Cruz. 2010:105:467-70.

27. King CH, Magak P, Salam EA, Ouma JH, Kariuki HC, Blanton RE, World Health Organization. Measuring morbidity in schistosomiasis mansoni: relationship between image pattern, portal vein diameter and portal branch thickness in large-scale surveys using new WHO coding guidelines for ultrasound in schistosomiasis. Tropical Med Int Health. 2003;8:109-17.

28. Richter J, Hatz C, Campagne G, Bergquist NR, Jenkins JM. Ultrasound in schistosomiasis: a practical guide to the standardised use of ultrasonography for the assessment of schistosomiasis-related morbidity. World Health Organization 2000. http://www.who.int/iris/handle/10665/ 66535.

29. Richter J. Evolution of schistosomiasis-induced pathology after therapy and interruption of exposure to schistosomes: a review of ultrasonographic studies. Acta Trop. 2000;77:111-31.

30. Doehring-Schwerdtfeger E, Kardorff R. Ultrasonography in schistosomiasis in Africa. Mem Inst Oswaldo Cruz. 1995:90:141-5.

31. Catalano OA, Sahani DV, Forcione DG, Czermak B, Liu CH, Soricelli A, et al. Biliary infections: spectrum of imaging findings and management. Radiographics. 2009;29:2059-80. 
32. Cheever AW, Warren KS. Hepatic blood flow in mice with acute hepatosplenic schistosomiasis mansoni. Trop Med Hyg. 1964;58:406-12.

33. Bosch J, Groszmann RJ, Shah VH. Evolution in the understanding of the pathophysiological basis of portal hypertension: how changes in paradigm are leading to successful new treatments. J Hepatol. 2015;62:5121-30.

34. Bloch EW, Wahab MFA, Warren KS. In vivo microscopic observations of the pathogenesis and pathophysiology of hepatosplenic schistosomiasis in the mouse liver. Am J Trop Med Hyg. 1972;21:546-57.

35. You H, McManus DP. Vaccines and diagnostics for zoonotic schistosomiasis japonica. Parasitology. 2015;142:271-89.

Submit your next manuscript to BioMed Central and we will help you at every step:

- We accept pre-submission inquiries

- Our selector tool helps you to find the most relevant journal

- We provide round the clock customer support

- Convenient online submission

- Thorough peer review

- Inclusion in PubMed and all major indexing services

- Maximum visibility for your research

Submit your manuscript at www.biomedcentral.com/submit 